\title{
BMJ Open Impact of serum uric acid levels on the clinical prognosis and severity of coronary artery disease in patients with acute coronary syndrome and hypertension after percutaneous coronary intervention: a prospective cohort study
}

\author{
Shiyu Zhang (D) , ${ }^{1}$ Xin Liu, ${ }^{1}$ Bingxue Song, ${ }^{1}$ Haichu Yu, ${ }^{1,2}$ Xiaodong Zhang, ${ }^{3}$ \\ Yanming Shao ${ }^{1}$
}

To cite: Zhang S, Liu X, Song B, et al. Impact of serum uric acid levels on the clinical prognosis and severity of coronary artery disease in patients with acute coronary syndrome and hypertension after percutaneous coronary intervention: a prospective cohort study. BMJ Open 2022;12:e052031. doi:10.1136/ bmjopen-2021-052031

- Prepublication history for this paper is available online To view these files, please visit the journal online (http://dx.doi. org/10.1136/bmjopen-2021052031).

Received 04 April 2021 Accepted 22 December 2021

(D) Check for updates

(C) Author(s) (or their employer(s)) 2022. Re-use permitted under CC BY-NC. No commercial re-use. See rights and permissions. Published by BMJ.

For numbered affiliations see end of article.

Correspondence to

Haichu Yu; haichuyu@163.com

\section{ABSTRACT}

Objective The impact of serum uric acid (sUA) levels on the clinical prognosis and severity of coronary artery disease in patients with acute coronary syndrome (ACS) and hypertension after percutaneous coronary intervention (PCl) is not fully clear. This study aimed to assess the association among sUA levels, clinical prognosis and severity of coronary artery disease in patients with ACS and hypertension after PCl.

Design In this prospective cohort study, we followed-up patients with ACS and hypertension after PCl for 1 year to explore the risk factors for 1 year total major adverse cardiovascular events (MACEs) and multivessel coronary artery disease, the dose-effect relationship among sUA levels, MACEs and severity of coronary artery disease and correlation between sUA levels and severity of coronary artery disease (Gensini score).

Setting/patients Several Chinese internists followedup 422 patients who were diagnosed with ACS and hypertension after $\mathrm{PCl}$ in a large tertiary hospital of Qingdao during the period from 1 June 2019 to 1 December 2019.

Outcome measures One-year follow-up MACEs results and coronary angiography results.

Results In the coronary angiography results, multivessel coronary artery disease $(28.5 \%$ vs $21.4 \%, p=0.006)$ and non-culprit lesion vascular occlusion $(11.7 \%$ vs $5.3 \%$, $\mathrm{p}=0.042$ ) were more common in the hyperuricaemia group, and the Gensini score $(26.69 \pm 13.46$ vs $17.66 \pm 10.57, p<0.001)$ was also higher. In the results of 1 -year MACEs, the incidence of all-cause mortality (3.5\% vs $2.5 \%, \mathrm{p}=0.037), \mathrm{PCl}$ or coronary artery bypass grafting therapy due to myocardial infarction or angina pectoris $(15.1 \%$ vs $7.6 \%, p=0.027)$, medication conservative therapy in hospital due to myocardial infarction or angina pectoris $(12.9 \%$ vs $6.7 \%, p=0.041)$ and total MACEs $(31.8 \%$ vs $16.9 \%, p=0.001)$ were higher in patients with hyperuricaemia. Univariate and multivariate logistic regression analysis models
Strengths and limitations of this study

- The present study was unique prospective study related to hyperuricaemia in specific patients with acute coronary syndrome and hypertension after percutaneous coronary intervention.

- The present study was a prospective, single-centre study with a small sample number which affected the representation of patients.

- The present study did not fully adjust for other potentially unknown confounding factors, which may have impacted the results.

- The present study did not rule out the effect of the patient's current medication such as hydrochlorothiazide diuretics on uric acid levels.

- The present study was an observational study, and related treatment such as serum uric acid-lowering therapy, was not implemented for these patients.

showed that hyperuricaemia was still an independent risk factor for total MACEs within 1 year $(\mathrm{OR}=2.618$, $95 \% \mathrm{Cl} 1.656$ to $4.139, \mathrm{p}<0.001 ; \mathrm{R}=1.920,95 \% \mathrm{Cl}$ 1.158 to $3.183, p=0.011$, respectively) and multivessel coronary artery disease $(\mathrm{OR}=2.140,95 \% \mathrm{Cl} 1.371$ to $3.342, p=0.001 ; 0 R=1.688,95 \% \mathrm{Cl} 1.051$ to 2.710 , $\mathrm{p}=0.030$, respectively) after adjusting for confounding factors. The severity of coronary artery disease (nonculprit lesion vascular occlusion $(4.7 \%$ vs $8.4 \%$ vs $9.6 \%$ vs $16.2 \%, p=0.041$ ); multivessel coronary artery disease ( $17.9 \%$ vs $22.4 \%$ vs $29.8 \%$ vs $35.2 \%, p=0.022$ ); Gensini score $(16.96 \pm 10.35$ vs $19.31 \pm 10.63$ vs $26.12 \pm 11.48$ vs $33.33 \pm 14.01, p<0.001)$ ) and the incidence of total MACEs $(13.2 \%$ vs $14.2 \%$ vs $34.6 \%$ vs $41 \%, p<0.001)$ increased significantly with the sUA levels increasing. Further, the Gensini score was positively correlated with uric acid levels $(r=0.515, p<0.001)$. 
Conclusions Hyperuricaemia is an independent risk factor for 1-year total MACEs and multivessel coronary artery disease in patients with ACS and hypertension after $\mathrm{PCl}$.

\section{INTRODUCTION}

At present, cardiovascular diseases (CVDs) have a high incidence and high fatality rate worldwide. Acute coronary syndrome (ACS) is one of the main CVDs, which includes unstable angina pectoris, non-ST segment elevation myocardial infarction (NSTEMI) and ST segment elevation myocardial infarction (STEMI). ${ }^{1}$ Although the current treatment strategies of ACS are continuously optimised and upgraded, its incidence has remained extremely high in recent years. ${ }^{2}$ In the treatment and prevention of ACS, it is urgent to discover and control the risk factors for ACS in a timely manner and the inter-relation and predictive value for the prognosis of ACS-related risk factors require further research and discussion. Hypertension is one of the most important risk factors for ACS. Hypertension is characterised by a high prevalence, high morbidity and high fatality rate, causing damage to important organs such as the heart, brain and kidney, seriously threatening human health and increasing the burden on families and society. ${ }^{34}$ Therefore, patients with ACS and hypertension need to pay more attention to the risk factors to improve prognosis. Numerous studies presented that serum uric acid (sUA) was an independent risk factor for hypertension and ACS and that sUA had a complex relationship with hypertension and ACS. ${ }^{5-12}$ But the association among sUA levels, coronary heart disease (CHD) and hypertension is still controversial. A cross-sectional populationbased study showed hyperuricaemia was closely related to CHD-related mortality irrespective of sex. Another relative study showed that the risk of non-fatal myocardial infarction increased significantly with the levels of uric acid increasing. And subgroup analysis suggested that uric acid was more closely related to myocardial infarction in female patients than male patients. ${ }^{13}$ However, several studies failed to show that relative association. A recent study which included 231 patients with chronic coronary syndromes showed the absence of correlation between sUA and coronary arteries disease. ${ }^{14}$ And Framingham Study which included 6763 patients followed-up for 117376 person-years showed sUA was no longer associated with CHD events and cardiovascular mortality after adjustment for related risk factors. ${ }^{15}$ In addition, the relationship between uric acid and hypertension has always been a hot topic of discussion. Previous studies showed that hyperuricaemia was common in patients with primary hypertension. And they influenced each other during the development process. ${ }^{16}$ Even uric acidlowering drugs can be used to prevent the increase of blood pressure in patients with primary hypertension. ${ }^{17}$ Other studies suggested high sUA levels not only were closely related to the severity of coronary artery disease, ${ }^{18}$ but also affected its prognosis. Relevant clinical studies presented that hyperuricaemia was an independent risk factor for poor prognosis in patients with chronic heart failure. ${ }^{19}$ In addition, hyperuricaemia was highly correlated with the occurrence of future cardiovascular events after percutaneous coronary intervention (PCI) in patients with CHD. ${ }^{20}{ }^{21}$ In view of the contradictory role of sUA in CHD and hypertension, when clinical research related to sUA are conducted, it is necessary to consider mutual potential impact among sUA, CHD and hypertension. However, there are few studies commonly involving uric acid, CHD and hypertension currently. Therefore this study included patients with ACS and hypertension after PCI to further explore the relationship among sUA levels, clinical prognosis and severity of coronary artery disease.

\section{METHODS}

\section{Study design and patients}

The present study was a prospective cohort study with 422 patients who were diagnosed with ACS and hypertension after PCI in the Affiliated Hospital of Qingdao University during the period from 1 June 2019 to 1 December 2019. Several Chinese internists followed-up them for 1 year. The prospective study complied with the Declaration of Helsinki and was approved.

\section{Patient and public involvement}

No patient involved.

\section{Grouping and definitions}

The study population was divided into two groups according to the presence or absence of hyperuricaemia.

Hyperuricaemia was defined as sUA levels of $>333$ $\mu \mathrm{mol} / \mathrm{L}(>5.6 \mathrm{mg} / \mathrm{dL}){ }^{22}$

Hypertension diagnosis was made according to the standard definitions of the 2020 International Society of Hypertension Global Hypertension Practice Guidelines. ${ }^{23}$

ACS diagnosis was made according to the standard definitions of the American College of Cardiology. ${ }^{24}$

Major adverse cardiovascular events (MACEs) were defined as all-cause death, PCI or coronary artery bypass grafting (CABG) therapy due to myocardial infarction or angina pectoris as well as medication conservative therapy in hospital due to myocardial infarction or angina pectoris.

Multivessel coronary artery disease referred to coronary angiography showing that coronary stenosis of two or more vessels was $\geq 75 \%$, and left main coronary artery disease was defined as multivessel coronary artery disease.

Non-culprit lesion vessel occlusion referred to the occurrence of calcification and chronic occlusive disease of coronary arteries that were not related to the disease.

The Gensini score was calculated using the scoring schema previously defined by Gensini. ${ }^{25}$ 


\section{Data collection}

The following information was collected: demographic characteristics; heart rate, systolic blood pressure (SBP) and diastolic blood pressure measured during hospitalisation; body weight and height measured during hospitalisation; calculated body mass index (BMI); history of smoking, drinking or diabetes; laboratory parameters (total cholesterol, triglycerides, low-density lipoprotein cholesterol (LDL-C), high-density lipoprotein cholesterol (HDL-C), aspartate aminotransferase, alanine aminotransferase, creatinine, calculated glomerular filtration rate (GFR), N-terminal prohormone of brain natriuretic peptide (NT-Pro BNP) and troponin I (TnI)); drugs using in treatment; echocardiography results; coronary angiography results during hospitalisation; MACEs results during the 1-year follow-up period.

\section{Follow-up}

Patients were followed-up through outpatient clinics and telephone calls for up to 1 year. During the 1-year follow-up period, 27 patients (13 patients in the normal sUA group and 14 patients in the hyperuricaemia group) were dropped out of the study due to loss of follow-up (changing of mobile phone number, moving due to special reasons and so on) or other reasons.

\section{Statistical analysis}

SPSS V.25.0 (IBM) was used for statistical analysis. Continuous variables are presented as means $\pm \mathrm{SD}$ or as medians and IQRs according to their distribution as determined by the Kolmogorov-Smirnov test. Comparisons between groups were performed with Student's t-test or nonparametric tests when as appropriate. Categorical variables are reported as percentages and were compared with the $\chi^{2}$ test. Univariate and multivariate logistic regression models were used to explore the risk factors for 1-year total MACEs and multivessel coronary artery disease. Multivariate logistic regression analysis was applied with the risk factors defined by univariate analysis with $\mathrm{p}<0.05$. Estimates of ORs and their 95\% CIs were reported. Correlation analysis adopted Spearman correlation analysis. $\mathrm{P}<0.05$ was considered statistically significant.

\section{RESULTS}

\section{Baseline characteristics of the patients}

A total of 422 participants were included, and there were $131(31 \%)$ patients in the hyperuricaemia group and $291(69 \%)$ patients in the normal sUA group. The characteristics of each group are shown in table 1 . In the general clinical data, the proportion of women, BMI, current smoking, current drinking, triglycerides, creatinine, GFR and TnI were higher in patients with hyperuricaemia. Diastolic blood pressure and HDL-C were lower in patients with hyperuricaemia. All patients registered took dual antiplatelet drugs. Our results showed no significant difference between two groups in dual antiplatelet therapy, lipid-lowering therapy, vasodilator therapy, heart rate-controlling therapy, diuretic therapy, antihypertensive therapy and so on.

\section{Coronary angiography results of the patients}

The results of coronary angiography of each group are shown in table 2. Multivessel coronary artery disease and non-culprit lesion vessel occlusion were more common in the hyperuricaemia group, and the Gensini score was higher in the hyperuricaemia group.

\section{One-year follow-up MACEs}

The results of the 1-year follow-up MACEs of each group are shown in table 2. The 27 patients were dropped out of the study due to loss of follow-up or other reasons. The 108 participants happened MACEs (sUA normal $(\mathrm{n}=20)$ and hyperuricaemia $(\mathrm{n}=88))$. The incidence of all-cause mortality, PCI or CABG therapy due to myocardial infarction or angina pectoris, medication conservative therapy in hospital due to myocardial infarction or angina pectoris and total MACEs were higher in patients with hyperuricaemia.

\section{Univariate and multivariate logistic regression analysis of 1-year follow-up MACEs}

The univariate and multivariate logistic regression analysis of the 1-year follow-up MACEs models are shown in table 3. Hyperuricaemia was an independent risk factor for 1-year total MACEs after adjustment for confounding variables (sex, age, BMI, SBP, GFR, current smoking, diabetes mellitus, total cholesterol, triglycerides, LDL-C, NT-pro BNP, TnI, left ventricular ejection fraction (LVEF), Gensini score, multivessel coronary lesions, nonculprit lesion vessel occlusion). The table 3 only showed the risk factors defined by univariate analysis with $\mathrm{p}<0.05$.

\section{Univariate and multivariate logistic regression analysis of the multivessel coronary artery disease}

The univariate and multivariate logistic regression analysis of the multivessel coronary artery disease models are shown in table 4. Hyperuricaemia was an independent risk factor for multivessel coronary artery disease after adjustment for confounding variables(sex, age, SBP, GFR, BMI, current smoking, diabetes mellitus, total cholesterol, triglycerides, LDL-C, TnI, NT-pro BNP, LVEF\%, nonculprit lesion vessel occlusion, Gensini score, multivessel coronary lesions). The table 4 only showed the risk factors defined by univariate analysis with $\mathrm{p}<0.05$.

\section{Dose-effect relationship among sUA levels, clinical prognosis} and severity of coronary artery disease

The sUA levels of all patients were grouped according to the IQR to investigate the dose-effect relationship associations among sUA levels, clinical prognosis and severity of coronary artery disease (table 5). As the levels of sUA increased, the severity of coronary artery disease (non-culprit lesion vessel occlusion; multivessel coronary artery disease; and Gensini score) increased. Further, the Gensini score was positively correlated with uric acid levels (figure 1). The figure is provided in online. In 
Table 1 Baseline characteristics of patients with normal sUA levels or hyperuricaemia

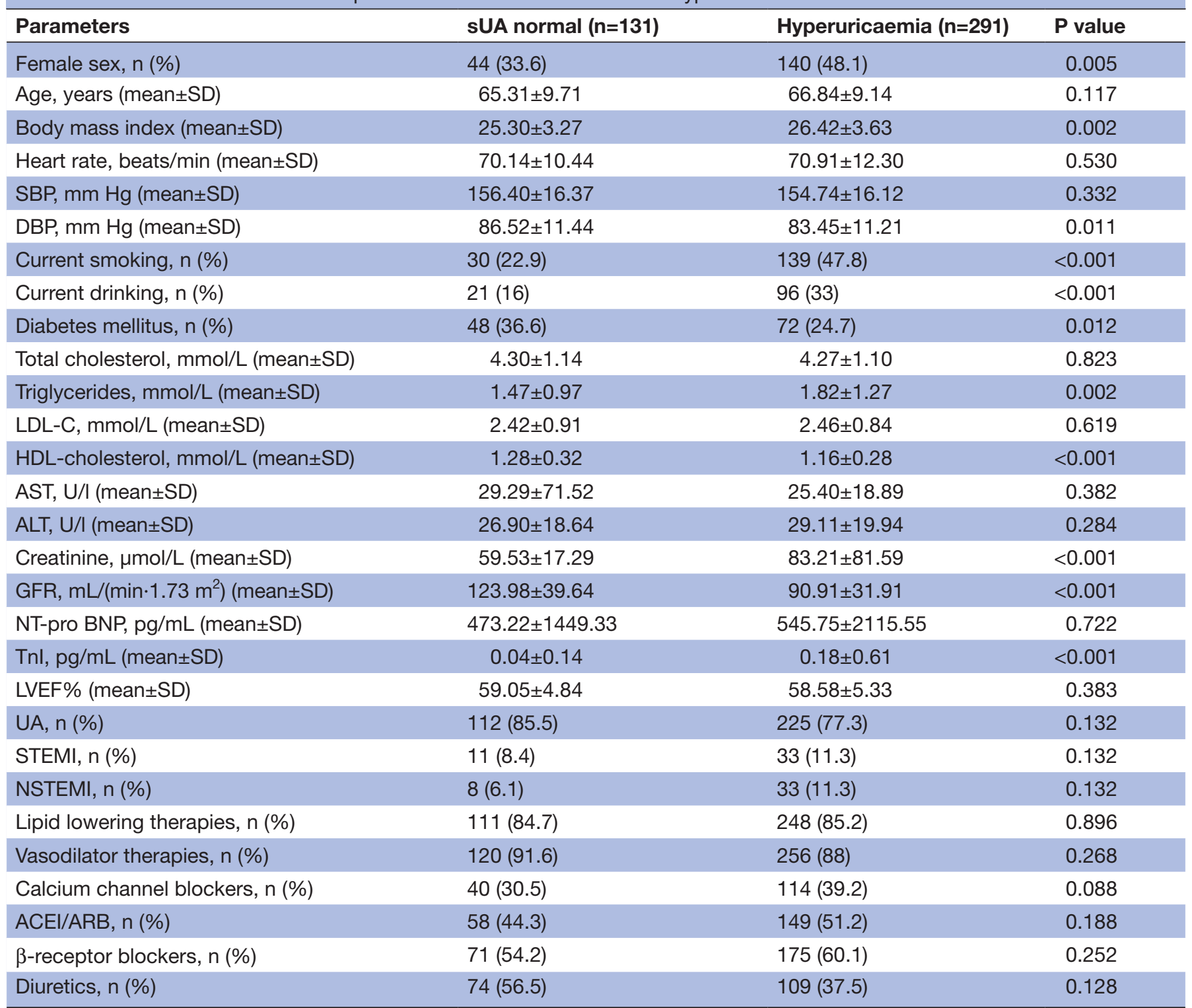

ACEI, ACE inhibitor; ALT, alanine aminotransferase; ARB, angiotensin receptor inhibitor; AST, aspartate aminotransferase; NT-pro BNP, Nterminal prohormone of brain natriuretic peptide; DBP, diastolic blood pressure; GFR, glomerular filtration rate; HDL, high density lipoprotein; LDL, low-density lipoprotein; LVEF, left ventricular ejection fraction; NSTEMI, non-ST segment elevation myocardial infarction; SBP, systolic blood pressure; STEMI, ST segment elevation myocardial infarction; sUA, serum uric acid; Tnl, troponin I; UA, unstable angina pectoris.

addition, the incidence of 1-year follow-up total MACEs also increased significantly with the sUA levels increasing.

\section{DISCUSSION}

This study demonstrated that hyperuricaemia was closely related to the incidence of 1-year MACEs and was an independent risk factor for 1-year total MACEs in patients with ACS and hypertension after PCI. To a certain extent, high sUA levels can reflect the clinical prognosis of these patients. In addition, we found that hyperuricaemia was closely related to the severity of coronary artery disease, and was an independent risk factor for multivessel coronary artery disease in patients with ACS and hypertension after PCI. Therefore, to a certain extent, high sUA level can also reflect the severity of coronary artery disease. As the levels of sUA increased, the incidence of 1-year follow-up total MACEs increased significantly and severity of coronary artery disease (non-culprit lesion vessel occlusion, multivessel coronary artery disease and Gensini score) increased. Further, the Gensini score was positively correlated with uric acid levels.

To date, the impact of sUA levels on the clinical prognosis and severity of coronary artery disease in patients with ACS and hypertension after PCI has not been fully clear. Therefore this study aimed to further explore the relationship among sUA levels, clinical prognosis and severity of coronary artery disease in patients with ACS and hypertension after PCI to provide a basis for reducing 
Table 2 Coronary angiography and 1-year follow-up MACEs results of patients with normal sUA levels or hyperuricaemia

\begin{tabular}{|c|c|c|c|}
\hline & sUA normal $(n=131)$ & Hyperuricaemia $(n=291)$ & $P$ value \\
\hline \multicolumn{4}{|l|}{ Coronary angiography } \\
\hline Number of patients & 131 & 291 & \\
\hline Non-culprit lesion vessel occlusion, n (\%) & $7(5.3)$ & $34(11.7)$ & 0.042 \\
\hline Multivessel coronary lesions, $\mathrm{n}(\%)$ & $28(21.4)$ & $83(28.5)$ & 0.006 \\
\hline Gensini score, $($ mean $\pm S D)$ & $17.66 \pm 10.57$ & $26.69 \pm 13.46$ & $<0.001$ \\
\hline \multicolumn{4}{|l|}{ One-year follow-up MACEs } \\
\hline Number of patients & 118 & 277 & \\
\hline All-cause mortality, n (\%) & $3(2.5)$ & $10(3.5)$ & 0.037 \\
\hline $\begin{array}{l}\mathrm{PCl} \text { or } \mathrm{CABG} \text { therapy due to myocardial infarction or } \\
\text { angina pectoris, } \mathrm{n}(\%)\end{array}$ & $9(7.6)$ & $42(15.1)$ & 0.027 \\
\hline $\begin{array}{l}\text { Medication conservative therapy in hospital due to } \\
\text { myocardial infarction or angina pectoris, } \mathrm{n}(\%)\end{array}$ & $8(6.7)$ & $36(12.9)$ & 0.041 \\
\hline Total MACEs, n (\%) & $20(16.9)$ & $88(31.8)$ & 0.001 \\
\hline
\end{tabular}

sUA, serum uric acid; $\mathrm{PCl}$, percutaneous coronary intervention; CABG, coronary artery bypass grafting; MACEs, major adverse cardiovascular events.

future cardiovascular events in patients with ACS and hypertension. Of course, the present study still had some limitations. First, the present study was a prospective, single-centre study with a small sample number, which affected the representation of patients. The number and scope of the included study population were relatively limited. Whether the study results can be equally suitable for patients in other region was not yet known. Second, the present study did not fully adjust for other potentially unknown confounding factors, which may have affected the results. Third, the present study did not yet rule out the effect of the patient's current medication such as hydrochlorothiazide diuretics on uric acid levels. Fourth, the present study was an observational study, and related treatment such as sUA-lowering therapy, was not implemented for these patients. Therefore, the relationship between the reduction in uric acid levels and the prognosis of patients with ACS and hypertension after PCI was not explored.

CVDs have the characteristics of a high fatality rate and high disability rate, especially CHD. It is of great significance to reduce the incidence of CVD and improve its prognosis. In recent years, hyperuricaemia has been suggested to be closely related to CVD. ${ }^{2627}$ Clinical studies related physiological and biochemical mechanisms have suggested that high sUA levels may increase oxidative stress, promote systemic inflammation, promote local inflammation, increase insulin resistance and increase other mechanisms to cause endothelial dysfunction and lead to the occurrence of CVDs, such as ACS. ${ }^{28-30}$ There are many clinical studies related to the effect of uric acid on the prognosis of patients with CHD. A retrospective study showed that high sUA levels at admission were independently correlated with death and other adverse cardiovascular events during hospitalisation. ${ }^{31}$ Another retrospective prognostic study presented that high sUA levels at admission indicated the higher mortality rate during hospitalisation, higher 30-day mortality rate and poor long-term (1-6 years) prognosis of patients with acute myocardial infarction (AMI) ${ }^{32}$ A meta-analysis of prognostic studies showed that high sUA levels at admission independently predicted worse short-term (during hospitalisation and 30 days after the occurrence of AMI) and medium or long-term ( 1 year or 6 months to 2 years after the occurrence of AMI) outcomes after AMI. ${ }^{33}$ Our study also confirmed that the long-term prognosis of

Table 3 Logistic regression analysis of related risk factors for total MACEs during 1-year follow-up

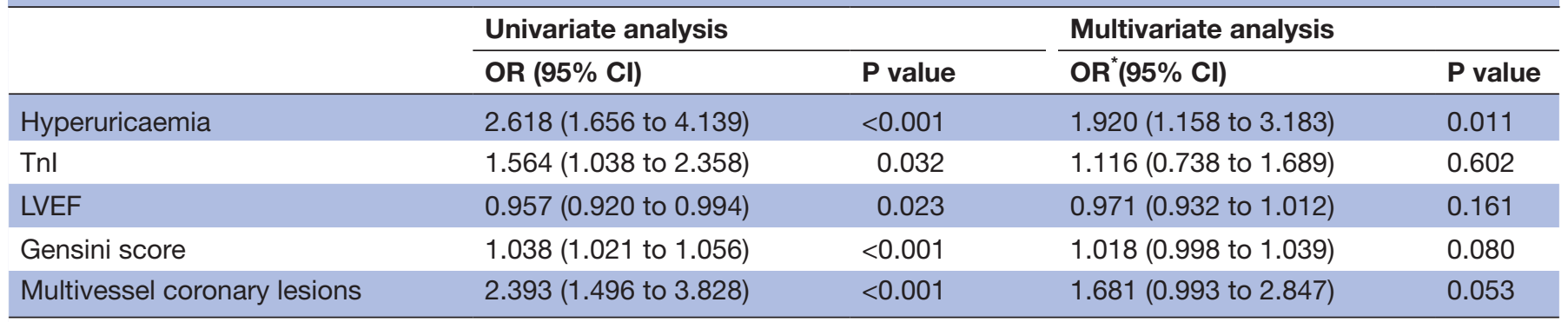

LVEF, left ventricular ejection fraction; MACEs, major adverse cardiovascular events; OR*, adjusted OR; Tnl, troponin I. 
Table 4 Logistic regression analysis of related risk factors for multivessel coronary lesions

\begin{tabular}{lllll} 
& \multicolumn{1}{l}{ Univariate analysis } & & Multivariate analysis & \\
\cline { 2 - 3 } \cline { 5 - 6 } & OR $\mathbf{( 9 5 \%}$ Cl) & P value & OR $^{*}$ (95\% Cl) & P value \\
\hline Hyperuricaemia & $2.140(1.371$ to 3.342$)$ & 0.001 & $1.688(1.051$ to 2.710$)$ & 0.030 \\
SBP & $1.020(1.006$ to 1.035$)$ & 0.005 & $1.025(1.010$ to 1.040$)$ & 0.001 \\
Current smoking & $1.748(1.151$ to 2.765$)$ & 0.010 & $1.941(1.218$ to 3.094$)$ & 0.005 \\
Creatinine & $1.006(1.001$ to 1.012$)$ & 0.023 & $1.006(1.001$ to 1.011$)$ & 0.021 \\
Tnl & $1.905(1.203$ to 3.016$)$ & 0.006 & $1.768(1.061$ to 2.946$)$ & 0.029 \\
\hline
\end{tabular}

$\mathrm{OR}^{\star}$, adjusted OR; SBP, systolic blood pressure; Tnl, troponin I.

patients with ACS in high uric acid levels group was worse (the incidence of MACEs increased significantly within 1 year). However, death and MACEs rarely happened during short-term follow-up (during hospitalisation and 30 days after AMI) in this study. Therefore, the impact of high uric acid levels on the short-term prognosis was not clear in our study. The Third National Health and Nutrition Examination Survey showed that sUA was independently associated with total mortality and cardiovascular mortality. As the levels of sUA increased, these risks significantly increased. ${ }^{34}$ Another retrospective cohort study also showed that hyperuricaemia was an independent risk factor for 1-year all-cause mortality in elderly female patients with ACS. ${ }^{35}$ Although these studies and others suggested that uric acid was closely related to the prognosis of $\mathrm{CHD}$, there were some studies that failed to draw these conclusions, which may be due to the poor sample size of the selected population, short follow-up time or selection of statistical methods. Therefore, although there are many related studies, the association between uric acid and the prognosis of CHD is still unclear, indicating the need for similar studies to be conducted. In addition, most of the subjects of these studies were patients with hyperuricaemia and ACS in the past, and the selected population type was relatively fixed. Further, it is necessary to pay particular attention to the prognosis of patients with ACS undergoing PCI. After PCI therapy, preventing coronary restenosis and myocardial infarction becomes more important, and these patients should pay more attention to the secondary prevention of the disease, controlling of cardiovascular risk factors, delaying of disease progression and increasing of life expectancy to further improve the prognosis. A retrospective analysis of 213 young adult patients ( $\leq 40$ years old) undergoing PCI showed that sUA levels were correlated with MACEs during long-term (930 days) follow-up in young patients with NSTEMI undergoing PCI. ${ }^{36}$ Another retrospective study presented that a high serum sUA level $(>5.6 \mathrm{mg} / \mathrm{dL})$ was associated with all-cause mortality in patients with ACS after PCI. ${ }^{37}$ Our study population was composed of mainly patients with ACS undergoing PCI, and the results were similar to the above research results.

However, previous studies identified that uric acid was also closely related to hypertension. The possible mechanisms of uric acid causing hypertension are as follows: sUA damages vascular endothelial cells by promoting oxidative stress reactions and causes vasomotor dysfunction by reducing the synthesis of nitric oxide. The sUA activates the renin-angiotensin-aldosterone system to lead to vascular contraction, vascular remodelling and water or sodium retention. ${ }^{8} 3839$ Hypertension is also the most important risk factor for CVD. In view of the inseparable and complex relationship among uric acid, hypertension and CHD, the effects of uric acid and hypertension on the prognosis of patients with CHD after PCI are unclear. For the studies about effects of uric acid on the prognosis of patients with CHD after PCI, it is necessary to consider whether patients also have the history of

Table 5 Dose-effect relationship among sUA levels, clinical prognosis and severity of coronary artery disease

$137 \sim 315 \mu \mathrm{mol} / \mathrm{L} \quad 316 \sim 387 \mu \mathrm{mol} / \mathrm{L} \quad 388 \sim 446 \mu \mathrm{mol} / \mathrm{L} \quad 347 \sim 659 \mu \mathrm{mol} / \mathrm{L} \quad P$ value

\begin{tabular}{|c|c|c|c|c|c|}
\hline \multicolumn{6}{|l|}{ Coronary angiography } \\
\hline $\begin{array}{l}\text { Non-culprit lesion vessel } \\
\text { occlusion, } \mathrm{n}(\%)\end{array}$ & $5(4.7)$ & $9(8.4)$ & $10(9.6)$ & $17(16.2)$ & 0.041 \\
\hline $\begin{array}{l}\text { Multi-vessel coronary artery } \\
\text { disease, } n(\%)\end{array}$ & $19(17.9)$ & $24(22.4)$ & $31(29.8)$ & 37 (35.2) & 0.022 \\
\hline Gensini score, $($ mean $\pm S D)$ & $16.96 \pm 10.35$ & $19.31 \pm 10.63$ & $26.12 \pm 11.48$ & $33.33 \pm 14.01$ & $<0.001$ \\
\hline \multicolumn{6}{|l|}{$\begin{array}{l}\text { One-year follow-up total } \\
\text { MACEs }\end{array}$} \\
\hline Total MACEs, n (\%) & $14(13.2)$ & $15(14.2)$ & $36(34.6)$ & $43(41)$ & $<0.001$ \\
\hline
\end{tabular}

MACEs, major adverse cardiovascular events; sUA, serum uric acid. 


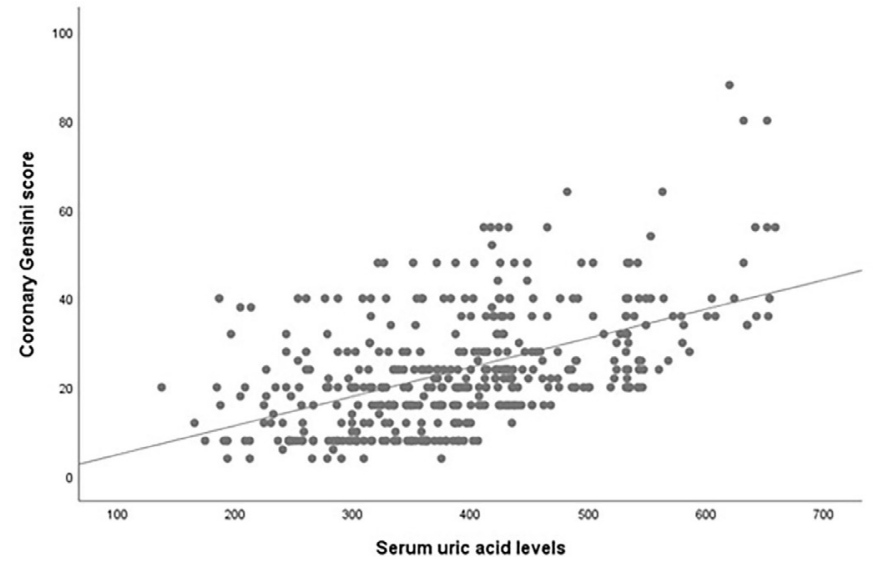

Figure 1 Correlation analysis of uric acid levels and the Gensini score.

hypertension. Therefore, our study population mainly focused on patients with ACS and hypertension after PCI. In this study, hyperuricaemia was an independent risk factor for 1-year total MACEs in these patients. As the levels of sUA increased, the incidence of 1-year follow-up total MACEs also significantly increased. These results fill the study gap in the prognosis of these patients and simultaneously provide a basis for further studies on the risk of cardiovascular events.

In addition, our study demonstrated that hyperuricaemia was closely related to the severity of coronary artery disease, and was an independent risk factor for multivessel coronary artery disease in patients with ACS and hypertension after PCI. As the levels of sUA increased, severity of coronary artery disease (non-culprit lesion vessel occlusion, multivessel coronary artery disease and Gensini score) increased. Further, the Gensini score was positively correlated with uric acid levels. The potential mechanisms of uric acid causing coronary lesions are as follows: sUA may result in atherosclerosis and blood vessel stenosis by causing LDL-C oxidative modification, promoting the release of inflammatory factors to stimulate vascular smooth muscle hyperplasia, activating platelets to promote intravascular thrombosis. ${ }^{28}$ In addition, the incidence of slow blood flow and no-reflow of the coronary artery increases. ${ }^{40}$ Previous studies on the relationship between uric acid levels and the severity of coronary artery disease found that the increase in sUA levels was closely related to the severity of coronary artery disease assessed by coronary angiography and that there was a positive correlation between sUA levels and the Gensini score of the severity of coronary artery disease. ${ }^{414}$ Our study further showed that the relationship between sUA levels and the Gensini score of the severity of coronary artery disease existed in patients with ACS and hypertension after PCI. Therefore, the levels of sUA still can be used as an indicator to assess the severity of coronary artery disease in these specific patients with ACS and hypertension after PCI.

In view of the metabolism levels of uric acid in different populations are different, and the effects on the prognosis of different populations are also discrepant it is necessary to conduct researches related uric acid in different populations. Therefore this study focuses on specific patients with ACS and hypertension after PCI to explore the impact of uric acid on the clinical prognosis and severity of coronary artery disease for the first time, helping to improve the prognosis of these patients. In addition, in view of limitations of the present study, multicentre, randomised, controlled and blinded studies with larger sample numbers and different populations are needed for further discussion.

\section{CONCLUSION}

Hyperuricaemia is an independent risk factor for 1-year total MACEs and multivessel coronary artery disease in patients with ACS and hypertension after PCI.

\section{Author affiliations}

${ }^{1}$ Cardiology, The Affiliated Hospital of Qingdao University, Qingdao, shandong, China ${ }^{2}$ Cardiology, The Affiliated Cardiovascular Hospital of Qingdao University, qingdao, shandong, china

${ }^{3}$ Cardiology, Jiaozhou Branch of Shanghai East Hospital,Tongji University, qingdao, shandong, china

Contributors SZ proposed study ideas and designed study plans. XZ and YS were responsible for collecting data and following-up patients for 1 year. $X L$ and $B S$ contributed significantly to analysis and manuscript preparation. SZ performed the data analyses and wrote the manuscript. HY was responsible for the revision of the final version of the article. HY was responsible for the overall content as the guarantor.

Funding The work was supported by Qingdao Municipal Science and Technology Bureau of China (Demonstration and Guidance Special Project of Science and Technology for the Favor of Public) (20-3-4-54-nsh).

Competing interests We have read and understood BMJ policy on declaration of interests and declare that we have no competing interests.

Patient consent for publication Not applicable.

Ethics approval Ethics approval was provided by the Ethical Review Board of Affiliated Hospital of Qingdao University(QYFYWZLL26229).

Provenance and peer review Not commissioned; externally peer reviewed.

Data availability statement № data are available. No data are available. The original data is owned by the hospital and I have no right to provide it to others or institutions.

Open access This is an open access article distributed in accordance with the Creative Commons Attribution Non Commercial (CC BY-NC 4.0) license, which permits others to distribute, remix, adapt, build upon this work non-commercially, and license their derivative works on different terms, provided the original work is properly cited, appropriate credit is given, any changes made indicated, and the use is non-commercial. See: http://creativecommons.org/licenses/by-nc/4.0/.

ORCID iD

Shiyu Zhang http://orcid.org/0000-0002-8802-1249

\section{REFERENCES}

1 Metkus T. Incidence, predictors and prognosis of respiratory support in non-ST segment elevation myocardial infarction. Eur Heart $J$ Acute Cardiovasc Care 2020.

$2 \mathrm{He} \mathrm{C}$, Lin P, Liu W, et al. Prognostic value of hyperuricemia in patients with acute coronary syndrome: a meta-analysis. Eur J Clin Invest 2019;49:e13074.

3 Whelton PK, Carey RM, Aronow WS, et al. 2017 ACC/AHA/AAPA ABC/ACPM/AGS/APhA/ASH/ASPC/NMA/PCNA guideline for the prevention, detection, evaluation, and management of high blood pressure in adults: Executive summary: a report of the American 
College of Cardiology/American heart association Task force on clinical practice guidelines. Hypertension 2018;71:1269-324.

4 Williams B, Mancia G, Spiering W, et al. 2018 ESC/ESH guidelines for the management of arterial hypertension. Eur Heart $J$ 2018:39:3021-104

5 Mallat SG, Al Kattar S, Tanios BY, et al. Hyperuricemia, hypertension, and chronic kidney disease: an emerging association. Curr Hypertens Rep 2016;18:74.

6 Cicero AFG, Rosticci M, Fogacci F, et al. High serum uric acid is associated to poorly controlled blood pressure and higher arterial stiffness in hypertensive subjects. Eur J Intern Med 2017;37:38-42.

7 Cao Z, Cheng Y, Li S, et al. Mediation of the effect of serum uric acid on the risk of developing hypertension: a population-based cohort study. J Transl Med 2019;17:202.

8 Biscaglia S, Ceconi C, Malagù M, et al. Uric acid and coronary artery disease: an elusive link deserving further attention. Int $J$ Cardiol 2016;213:28-32.

9 Zhang J-wei, He L-jie, Cao S-jun, et al. Association of serum uric acid and coronary artery disease in premenopausal women. PLOS One 2014;9:e106130.

10 Loeffler LF, Navas-Acien A, Brady TM, et al. Uric acid level and elevated blood pressure in US adolescents: National health and nutrition examination survey, 1999-2006. Hypertension 2012;59:811-7.

11 Fan Y, Wei F, Lang Y, et al. Losartan treatment for hypertensive patients with hyperuricaemia in Chinese population: a meta-analysis. $J$ Hypertens 2015;33:681-9.

12 De Becker B, Borghi C, Burnier M, et al. Uric acid and hypertension: a focused review and practical recommendations. $J$ Hypertens 2019:37:878-83.

13 Saito Y, Tanaka A, Node K, et al. Uric acid and cardiovascular disease: a clinical review. J Cardiol 2021;78:51-7.

14 Maloberti A, Bossi I, Tassistro E, et al. Uric acid in chronic coronary syndromes: relationship with coronary artery disease severity and left ventricular diastolic parameter. Nutr Metab Cardiovasc Dis 2021;31:1501-8.

15 Culleton BF, Larson MG, Kannel WB, et al. Serum uric acid and risk for cardiovascular disease and death: the Framingham heart study. Ann Intern Med 1999;131:7-13.

16 Zhang S, Wang Y, Cheng J, et al. Hyperuricemia and cardiovascular disease. Curr Pharm Des 2019;25:700-9.

17 Zeng F, Huang R, Lu Y, et al. Association of anti-hyperuricemia treatment and prevalent cardiovascular disease in hypertensive patients. Arch Med Sci 2020;16:545-50.

18 Gaubert M, Marlinge M, Alessandrini M, et al. Uric acid levels are associated with endothelial dysfunction and severity of coronary atherosclerosis during a first episode of acute coronary syndrome. Purinergic Signal 2018;14:191-9.

19 Li M, Hou W, Zhang X, et al. Hyperuricemia and risk of stroke: a systematic review and meta-analysis of prospective studies. Atherosclerosis 2014;232:265-70.

20 Spoon DB, Lerman A, Rule AD, et al. The association of serum uric acid levels with outcomes following percutaneous coronary intervention. J Interv Cardiol 2010:23:277-83.

21 Lim SS, Yang Y-L, Chen S-C, et al. Association of variability in uric acid and future clinical outcomes of patient with coronary artery disease undergoing percutaneous coronary intervention. Atherosclerosis 2020;297:40-6.

22 Maloberti A, Giannattasio C, Bombelli M, et al. Hyperuricemia and risk of cardiovascular outcomes: the experience of the URRAH (uric acid right for heart health) project. High Blood Press Cardiovasc Prev 2020;27:121-8.
23 Unger T, Borghi C, Charchar F, et al. 2020 International Society of hypertension global hypertension practice guidelines. J Hypertens 2020;38:982-1004.

24 Amsterdam EA, Wenger NK, Brindis RG. 2014 AHA/ACC guideline for the management of patients with non-ST-elevation acute coronary syndromes: a report of the American College of Cardiology/American heart association Task force on practice guidelines. Circulation 20142014;130:e344-426.

25 Gensini GG. A more meaningful scoring system for determining the severity of coronary heart disease. Am J Cardiol 1983;51:606.

26 Bove M, Cicero AFG, Veronesi M, et al. An evidence-based review on urate-lowering treatments: implications for optimal treatment of chronic hyperuricemia. Vasc Health Risk Manag 2017;13:23-8.

27 Borghi C, Desideri G. Urate-Lowering drugs and prevention of cardiovascular disease: the emerging role of xanthine oxidase inhibition. Hypertension 2016;67:496-8.

28 Ndrepepa G. Uric acid and cardiovascular disease. Clin Chim Acta 2018;484:150-63.

29 Li P, Zhang L, Zhang M, et al. Uric acid enhances PKC-dependent eNOS phosphorylation and mediates cellular ER stress: a mechanism for uric acid-induced endothelial dysfunction. Int J Mol Med 2016;37:989-97.

30 Cai W, Duan X-M, Liu Y, et al. Uric acid induces endothelial dysfunction by activating the HMGB1/RAGE signaling pathway. Biomed Res Int 2017;2017:1-11.

31 Centola M, Maloberti A, Castini D, et al. Impact of admission serum acid uric levels on in-hospital outcomes in patients with acute coronary syndrome. Eur J Intern Med 2020;82:62-7.

32 Car S, Trkulja V. Higher serum uric acid on admission is associated with higher short-term mortality and poorer long-term survival after myocardial infarction: retrospective prognostic study. Croat Med J 2009:50:559-66.

33 Trkulja V, Car S. On-admission serum uric acid predicts outcomes after acute myocardial infarction: systematic review and metaanalysis of prognostic studies. Croat Med J 2012;53:162-72.

34 Stack AG, Hanley A, Casserly LF, et al. Independent and conjoint associations of gout and hyperuricaemia with total and cardiovascular mortality. QJM 2013;106:647-58.

35 Tai S, Li X, Zhu Z, et al. Hyperuricemia is a risk factor for oneyear overall survival in elderly female patients with acute coronary syndrome. Cardiovasc Ther 2020;2020:1-10.

36 Canga $Y$, Emre A, Karatas MB, et al. Prognostic value of serum uric acid levels in patients with non-STEMI undergoing percutaneous coronary intervention. Herz 2020;45:389-96.

$37 \mathrm{Ye} \mathrm{Z}$, Lu H, Long M, et al. Baseline serum uric acid levels are associated with all-cause mortality in acute coronary syndrome patients after percutaneous coronary intervention. Dis Markers 2018:2018:1-8.

38 Richette P, Perez-Ruiz F, Doherty M, et al. Improving cardiovascular and renal outcomes in gout: what should we target? Nat Rev Rheumatol 2014;10:654-61.

39 Mishima M, Hamada T, Maharani N, et al. Effects of uric acid on the NO production of HUVECs and its restoration by urate lowering agents. Drug Res 2016;66:270-4.

40 Brosh D, Assali AR, Mager A, et al. Effect of no-reflow during primary percutaneous coronary intervention for acute myocardial infarction on six-month mortality. Am J Cardiol 2007;99:442-5.

41 Kumbhalkar S, Deotale R. Association between serum uric acid level with presence and severity of coronary artery disease. J Assoc Physicians India 2019;67:29-32.

$42 \mathrm{Yu}$ J, Han J, Mao J, et al. Association between serum uric acid level and the severity of coronary artery disease in patients with obstructive coronary artery disease. Chin Med J 2014;127:1039-45. 